\title{
EXTREME HAMILTONIAN CIRCUITS. RESOLUTION OF THE CONVEX-EVEN CASE
}

\author{
LOUIS V. QUINTAS AND FRED SUPNICK
}

Let $r$ noncollinear points in the Euclidean plane fall on the boundary $B$ of their convex hull. It is known that the shortest polygon having these points as vertices coincides with $B$. In [1] the ordering of these points which yields the longest polygon is obtained for the case where $r$ is odd. In this paper the even case is resolved.

THEOREM. Let $\Sigma$ denote a set of 2 n noncollinear coplanar points which fall on the boundary $B$ of their convex hull and let $P_{1}^{1}, P_{1}^{2}, \cdots, P_{1}^{n}$ denote any $n$ points of $\Sigma$ which are adjacent on $B$. Then, every longest polygon having precisely the points of $\Sigma$ as vertices is among the $n$ polygons $^{1}$

$$
\begin{array}{r}
{\left[\cdots P_{2 n-5}^{i} P_{5}^{i} P_{2 n-3}^{i} P_{3}^{i} P_{2 n-1}^{i} P_{1}^{i} P_{2 n}^{i} P_{2}^{i} P_{2 n-2}^{i} P_{4}^{i} P_{2 n-4}^{i} P_{6}^{i} \cdots\right]} \\
(i=1,2, \cdots, n)
\end{array}
$$

where for each $i$, starting with $P_{1}^{i}$ and traversing $B$ in a specified common direction the consecutive points of $\Sigma$ are labeled

$$
P_{1}^{i}, P_{2}^{i}, P_{3}^{i}, \cdots, P_{n}^{i}, P_{2 n}^{i}, P_{2 n-1}^{i}, P_{2 n-2}^{i}, \cdots, P_{n+1}^{i} .
$$

REMARK. The collinear case (odd or even) is a special case of Theorem III [2, p. 181].

Proof of the Theorem. Case I. Suppose no three points of $\Sigma$ are collinear. A line segment $D E$ with endpoints in $\Sigma$ is said to be of type $L_{k}(1 \leqq k \leqq n)$, if $D$ and $E$ are the endpoints of a polygonal subarc of $B$ having $k$ edges. For the cases where $2 n$ is equal to 2 or 4 , the Theorem is obviously true. Thus, in all that follows we assume $6 \leqq 2 n$.

Let $h=\left[R_{1} \cdots R_{2 n}\right]$ denote any polygon having the points of $\Sigma$ as vertices and having at least one edge $R_{i} R_{i+1}$ (subscripts reduced modulo $2 n$ ) of type $L_{k}$ with $1 \leqq k \leqq n-2$. It will be shown that, in this case, it is possible to construct a polygon longer than $h$. The vertices $R_{i}$ and $R_{i+1}$ define the following partition of $B: B_{1} \cup B_{2} \cup\left\{R_{i}, R_{i+1}\right\}$, where $B_{1}$ is the component of $B-\left\{R_{i}, R_{i+1}\right\}$ containing exactly $k-1$ points of $\Sigma$. We first show that there are at least three edges of $h$ having both vertices in $B_{2}$ (only two of these edges will be used in

Presented to the Society, April 20, 1964; received by the editors February 25, 1964.

1 The symbols for polygons are to be considered cyclic and symmetric. 
what follows). There are at most $2(k-1)+2$ edges of $h$ incident to the vertices in $B_{1} \cup\left\{R_{i}, R_{i+1}\right\}$ which terminate at vertices in $B_{2}$ and there are $2 n-(k-1)-2$ points of $\Sigma$ in $B_{2}$. Thus, there are at least the following number of edges of $h$ which have both vertices in $B_{2}$ :

$$
N=\frac{2(2 n-(k-1)-2)-(2(k-1)+2)}{2}=2 n-2 k-1 .
$$

Since $k \leqq n-2$, we have $N \geqq 2 n-2(n-2)-1=3$.

In order to describe how $h$ can be transformed into a polygon of strictly greater length we introduce the following notation. The symbol $\left[V_{1} \cdots V_{i-1}\left(V_{i} \cdots V_{j}\right) V_{j+1} \cdots V_{r}\right]$ will denote the polygon $\left[V_{1} \cdots V_{i-1} V_{j} V_{j-1} \cdots V_{i} V_{j+1} \cdots V_{r}\right]$ and the operation $[\cdots(\cdots) \cdots]$ will be referred to as an arcinversion (cf. [2, p. $180])$. Throughout this paper, all edges are assumed to be closed. Let $P Q$ and $R S$ denote disjoint directed edges with endpoints in $\Sigma$ and $C$ the boundary of the convex hull of $\{P, Q, R, S\}$. We shall say that $P Q$ and $R S$ have the same or opposite $C$-sense accordingly as they agree or conflict in inducing an orientation of $C$. We note that an oriented polygon having two edges with the same $C$-sense can be transformed into a longer polygon by an arcinversion.

Let $R_{j} R_{j+1}$ and $R_{k} R_{k+1}$ denote two edges of $h$ each of which has both vertices in $B_{2}$ and let all edges of $h$ be directed so as to agree with a fixed orientation of $h$. Note that the (closed) edges $R_{j} R_{j+1}$ and $R_{k} R_{k+1}$ may intersect. The case where they are adjacent edges is not excluded. However, they are both disjoint from $R_{i} R_{i+1}$. Thus, we may speak of the $C$-sense of $R_{i} R_{i+1}$ with respect to $R_{j} R_{j+1}$ and $R_{k} R_{k+1}$ respectively. If $R_{i} R_{i+1}$ has the same $C$-sense as either $R_{j} R_{j+1}$ or $R_{k} R_{k+1}$, say $R_{j} R_{j+1}$, then the arcinversion $\left[\cdots R_{j}\left(R_{j+1} \cdots R_{i}\right) R_{i+1} \cdots\right]$ yields a polygon which is longer than $h$. If $R_{i} R_{i+1}$ has $C$-sense opposite to both $R_{j} R_{j+1}$ and $R_{k} R_{k+1}$, let $C^{\prime}$ denote the boundary of the convex hull of these three edges and $R$ that terminal point of $R_{j} R_{j+1}$ or $R_{k} R_{k+1}$ which is adjacent to $R_{i+1}$ on $C^{\prime}$. Suppose $R=R_{j+1}$ (if $R=R_{k+1}$ a completely analogous situation exists). Then, consider the edge $R_{j+1} R_{j+2}$ and the partition of $B$ into the two components $B_{3}$ and $B-B_{3}$, where $B_{3}$ is the closed subarc of $B-\left\{R_{j}\right\}$ with endpoints $R_{i}$ and $R_{j+1}$. Note that $R_{j+2}$ is not necessarily in $B_{2}$. Then, either (i) $R_{j+2}$ is in $B_{3}, R_{j+1} R_{j+2}$ has the same $C$-sense as $R_{k} R_{k+1}$, and the arcinversion $\left[\cdots R_{j+1}\left(R_{j+2} \cdots R_{k}\right) R_{k+1} \cdots\right]$ yields a polygon which is longer than $h$ or (ii) $R_{j+2}$ is in $B-B_{3}, R_{j+1} R_{j+2}$ has the same $C$-sense as $R_{i} R_{i+1}$, and the arcinversion $\left[\cdots R_{j+1}\left(R_{j+2} \cdots R_{i}\right) R_{i+1} \cdots\right]$ yields a polygon which is longer than $h$. Therefore, a longest polygon cannot contain any edges of type $L_{k}$ with $1 \leqq k \leqq n-2$. 
Consider now a polygon $h^{\prime}$ which consists entirely of edges of type $L_{n-1}$. Note that for $2 n$ vertices such a polygon exists only if $n-1$ and $2 n$ are relatively prime, that is, only if $n$ is even. The polygon $h^{\prime}$ is represented by

$$
\left[P_{1}^{1} P_{n}^{1} P_{n+2}^{1} P_{n-2}^{1} P_{n+4}^{1} \cdots P_{2 n}^{1} P_{n+1}^{1} P_{n-1}^{1} P_{n+3}^{1} P_{n-3}^{1} \cdots\right]
$$

where the vertices are labeled as indicated in (2) with $i=1$. The arcinversion $\left[P_{1}^{1}\left(P_{n}^{1} \cdots P_{2 n}^{1}\right) P_{n+1}^{1} \cdots\right]$ applied to (3) yields a polygon which is longer than $h^{\prime}$. Specifically, this arcinversion yields the polygon (1) with $i=1$, which contains two edges of type $L_{n}$ and $2 n-2$ edges of type $L_{n-1}$.

To complete the proof of Case I it remains to show: if $w$ is any polygon containing at least one edge of type $L_{n}$ and having all other edges of type $L_{n}$ or of type $L_{n-1}$, then $w$ is one of the $n$ polygons indicated in the statement of the theorem. We shall refer to these $n$ polygons as $\beta$-polygons.

We select any edge of type $L_{n}$ in $w$, label it $W_{1} W_{2 n}$, and call it the first edge of $w$. For the second edge we select the other edge incident to $W_{2 n}$, label it $W_{2 n} W_{2}$ and note that it is of type $L_{n-1}$. The rest of the vertices of $w$ can now be labeled

$$
W_{1}, W_{2}, W_{3}, \cdots, W_{n}, W_{2 n}, W_{2 n-1}, W_{2 n-2}, \cdots, W_{n+1}
$$

in cyclic order around the boundary $B$ of their convex hull in a unique way compatible with the three vertices labeled thus far. Orienting $w$ by $W_{1} W_{2 n}$, we note that all odd numbered edges will terminate in vertices having subscript greater than $n$, and all even numbered edges will terminate in vertices having subscript less than or equal to $n$. Thus, edge $2 n$ must be $W_{2 n-1} W_{1}$. Since $6 \leqq 2 n$, the third edge of $w$ must be the edge $W_{2} W_{2 n-2}$ and edge $2 n-1$ must be the edge $W_{3} W_{2 n-1}$. Both of these edges are of type $L_{n-1}$.

The fourth edge must either be of type $L_{n}$ and completes $w$ (if and only if $n=3$ ), or must be the edge $W_{2 n-2} W_{4}$. If $n>3$, then edge $2 n-2$ must be the edge $W_{2 n-3} W_{3}$.

We proceed in this manner, repeating the above argument until the vertices $W_{n}$ and $W_{n+1}$ are joined by an edge of type $L_{n}$. This edge completes $w$ which is seen to be of the form (1) with $W$ in place of $P^{i}$.

If $W_{1} W_{2}$ and $P_{1}^{1} P_{2}^{1}$ induce the same orientation of $B$ and $W_{1}=P_{1}^{t}$ for some $i \in\{1,2, \cdots, n\}$, then $w$ is a $\beta$-polygon.

Suppose that $W_{1} W_{2}$ and $P_{1}^{1} P_{2}^{1}$ induce the same orientation of $B$ but $W_{1} \neq P_{1}^{i}$ for some $i \in\{1,2, \cdots, n\}$. Then,

$$
W_{i}^{\prime}=W_{2 n+1-i} \quad(i=1,2, \cdots, 2 n)
$$


defines a relabeling of the vertices of $w$ such that $w$ is of the form (1) with $W^{\prime}$ in place of $P^{i}, W_{1}^{\prime} W_{2}^{\prime}$ and $P_{1}^{1} P_{2}^{1}$ induce the same orientation of $B$, and $W_{1}^{\prime}=P_{1}^{i}$ for some $i \in\{1,2, \cdots, n\}$. Thus, $w$ is a $\beta$ polygon.

Suppose that $W_{1} W_{2}$ and $P_{1}^{1} P_{2}^{1}$ induce opposite orientations of $B$. In this case,

$$
W_{i}^{\prime}= \begin{cases}W_{n+i} & (i=1,2, \cdots, n), \\ W_{i-n} & (i=n+1, n+2, \cdots, 2 n)\end{cases}
$$

defines a relabeling of the vertices of $w$ such that $w$ is of the form (1) with $W^{\prime}$ in place of $P^{i}$, and $W_{1}^{\prime} W_{2}^{\prime}$ and $P_{1}^{1} P_{2}^{1}$ induce the same orientation of $B$. Thus, by the preceding two paragraphs, $w$ is a $\beta$-polygon.

Case II. Suppose $B$ has support lines passing through at least three points of $\Sigma$. Let $X$ be a point in the interior of the convex hull of $\Sigma$ and $B(t)(0 \leqq t<1)$ be a family of strongly convex curves circumscribing $B$ and converging to $B$ as $t$ approaches 1 . Let $P_{\jmath}^{i}(t)$ be the intersection of $B(t)$ with the ray emanating from $X$ and passing through $P_{j}^{i}(1 \leqq j \leqq 2 n ; 1 \leqq i \leqq n)$ and let $\Sigma(t) \equiv\left\{P_{j}^{i}(t)\right\}$. Then, for each $t(0 \leqq t<1)$ Case I implies that every longest polygon having the points of $\Sigma(t)$ as vertices is a $\beta(t)$-polygon. Now, for $t$ sufficiently close to 1 , a polygon having $P_{j}^{i}(t)(1 \leqq j \leqq 2 n ; i$ fixed) as vertices is arbitrarily close to the corresponding polygon having the $P_{j}^{i}$ 's as vertices. Therefore, every longest polygon with vertices in $B$ is a $\beta$-polygon.

REMARK. If the points of $\Sigma$ are evenly distributed on a circle, then the $n \beta$-polygons have the same length. On the other hand, sets $\Sigma$ (whose points all fall on the boundary of their convex hull) may be selected such that no two polygons having the points of $\Sigma$ as their vertices are equal in length.

\section{REFERENCES}

1. F. Supnick and L. V. Quintas, Extreme Hamiltonian circuits. Resolution of the convex-odd case, Proc. Amer. Math. Soc. 15 (1964), 454-456.

2. F. Supnick, Extreme Hamiltonian lines, Ann. of Math. (2) 66 (1957), 179-201.

ST. JoHN'S UNIVERSITY, NEW YoRK AND City College, New York 